\title{
TRANSFORMER RATIO ENHANCEMENT USING A RAMPED BUNCH TRAIN IN A COLLINEAR WAKEFIELD ACCELERATOR
}

\author{
J.G. Power ${ }^{\dagger}$, W. Gai, X. Sun, ANL, Argonne, IL, USA, 60439 \\ A. Kanareykin ${ }^{*}$ St. Petersburg Elec. Eng. Univ. 5 Prof. Popov St. Petersburg, 197376, Russia
}

\begin{abstract}
We present a practical method for achieving a transformer ratio $(R)$ greater than 2 with any collinear wakefield accelerator - i.e. with either plasma or structure based wakefield accelerators. It is known that the transformer ratio cannot generally be greater than 2 for a symmetric drive bunch in a collinear wakefield accelerator. However, using a ramped bunch train (RBT) where a train of $\mathrm{n}$ electron drive bunches, with increasing ('ramping') charge, one can achieve $R=2 n$ after the bunch train. We believe this method is feasible from an engineering standpoint. We describe a proof of principle experiment using an disk-loaded waveguide, of frequency $13.65 \mathrm{GHz}$, driven by a RBT of 4 electron bunches. We expect to achieve $R>6$ using 4 electron bunches. Details of the simulation and experimental design are presented.
\end{abstract}

\section{INTRODUCTION}

In general, the wakefield theorem [1] restricts the maximum accelerating field behind the drive bunch in a wakefield accelerator to be less than twice the maximum retarding field inside the drive bunch thus limiting the efficiency which can be obtained. One of the concepts central to the physics of wakefield acceleration is the transformer ratio, $R$, defined as $R=$ (Maximum energy gain behind the bunch)/(Maximum energy loss inside the drive bunch). For the case of a collinear drive and witness beam geometry device, $R$ is less than 2 except in a few special cases. For the purposes of this paper we only consider one regime where the wakefield theorem does not apply, namely, the use of an axially asymmetric charge distribution in the drive bunch. In this paper we review the RBT method and describe an experiment to be conducted at the Argonne Wakefield Accelerator (AWA).

\section{TRANSFORMER RATIO ENHANCEMENT}

Since the theoretical development of the transformer ratio enhancement is given elsewhere [4] we will not provide it here. In this section we give a brief historical account of the previous attempts to achieve $R>2$ and a summary of the RBT method.

\subsection{History}

Several schemes have been proposed to obtain $R>2$ in

†jpanl.gov collinear wakefield accelerators, but no experimental results have been obtained due to the inherent difficulties of these experiments. One of the more promising schemes [2] sends a single drive bunch, with an asymmetric axial current distribution (Fig. 1a) through a collinear wakefield accelerator. Simulations show that $R$ can be much greater than 2 for the triangular (ramped) bunch distribution as seen in the figure. Notice that most of the particles in the drive bunch experience the same decelerating wakefield, $W$, but the accelerating wakefield behind the bunch, $W^{+}$, is much larger. Using a similar idea, a second scheme tailors the profile of a train of drive bunches [3] into a triangular ramp (see dotted line in Fig. 1b) to produce $R>2$. In this later scheme, the individual bunches in the train are symmetric (e.g. gaussian) separated by a distance $d$. The charge is then ramped up such that

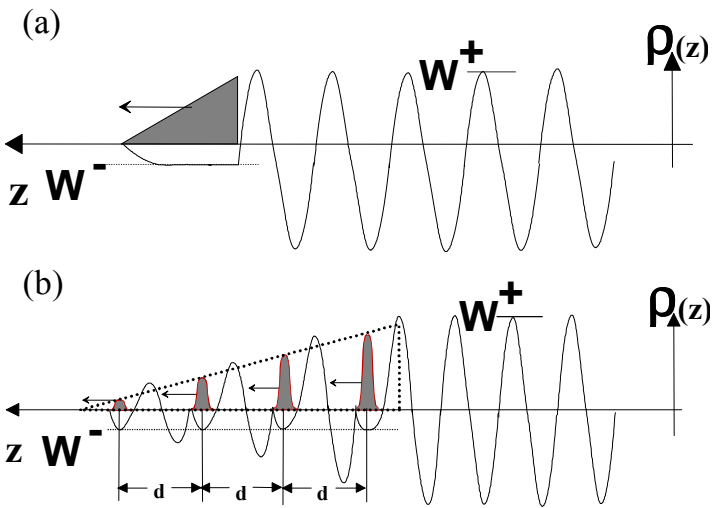

Figure 1: Two schemes that have been proposed to generate $R=W^{+} / W>>2$. The height of the shaded area, $\rho(z)$, represents the total amount of charge in the bunch at location $z$ while the solid, sine-like, line is the amplitude of the wakefield driven by the beam. (a) A single drive bunch with a triangular axial current distribution moving to the left. (b) A train of gaussian drive bunches with an overall triangular pattern of the train (see dotted line) moving to the left.

the first bunch in the train has the lowest charge and the last bunch the highest. From the figure we see that all four drive bunches in the drive train experience the same maximum decelerating field $W$ just like in the case of a single triangular ramped bunch. Thus, the fundamental condition for both of these schemes is that the trailing particles (bunches) in the drive bunch (train) are positioned in the accelerating phase of the leading particles (bunches) so that all the driving particles experience the same maximum decelerating field. 
The difficulty with the schemes that propose to use asymmetric axial current distribution to achieve $R>2$, arises from the lack of suitable techniques to tailor the axial distribution of the drive beam. In this paper we consider the later method, called, the 'ramped' bunch train (RBT) method of transformer ratio enhancement.

\subsection{Transformer Ratio and Acceleration Gradient for a Single bunch}

In order to design an RBT experiment, we must first understand the dependence of the transformer ratio and the peak acceleration gradient on a single electron bunch. Therefore, we recall [4] the dependence of the transformer ratio $\left(R_{0}\right)$ and the peak acceleration gradient $\left(W_{0}^{+}\right)$of a single bunch - where the subscript ' 0 ' denotes a single bunch.

As shown in Fig. 2, numerical simulations of a single drive bunch passing through a collinear wakefield accelerator show that $R_{0}$ and $W_{0}^{+}$are not maximum at the same value of normalized bunch length $\left(\sigma / \lambda_{0}\right)$.

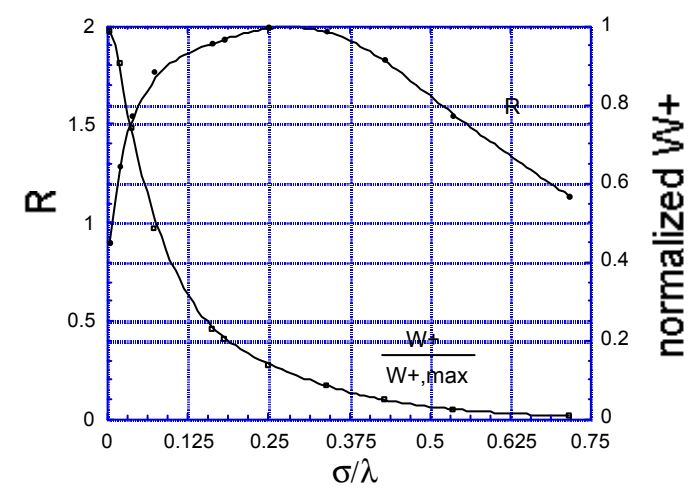

Figure 2: $\mathrm{R}$ is maximized when $\sigma / \lambda_{0} \sim 0.25$, while $\mathrm{W}^{+}$ is maximum as $\sigma / \lambda_{0} \rightarrow 0$.

\subsection{RBT Algorithm}

From [4] we have the following algorithm for creating the bunch train. Given the transformer ratio after the first $(n=0)$ bunch $\left(R_{0}\right)$ then the maximum transformer ratio that can be achieved after the $n$th bunch $\left(R_{n}\right)$ is,

$$
R_{n}=(n+1) R_{0}(n=0,1,2, \ldots N-1)
$$

This maximum enhancement of the transformer ratio can only be achieved if the following conditions are satisfied. The separation between the bunches is $1 / 2$ integer or,

$$
d=(m+1 / 2) \lambda_{0}(m=1,2, \ldots, N-1)
$$

where $\lambda_{0}$ is the fundamental wavelength of the structure. The charge ratio of the individual bunches within the train increases according to,

$$
Q_{n}=Q_{0}\left[n R_{0}+1\right] \quad(n=0,1,2, \ldots N-1)
$$

where $Q_{0}$ is the charge in the first drive bunch.

In addition to the requirements of spacing (Eqn. 2) and relative charge ratio (Eqn. 3) for the bunch train, there are also two requirements on the single bunch for obtaining maximal transformer ratio enhancement. The first condition is in addition to the work of [3]. The self-wake generated by the single bunch must be symmetric with respect to the center of the bunch. In other words, for a gaussian distribution, centered at $z=0$, the self-wake within the bunch must satisfy, $W_{0}^{-}(-z)=W_{0}^{-}(+z)$. If this 'symmetric, single wake' condition is not met, the selfwakefields of the trailing bunch will be phase shifted from the accelerating bucket of the leading bunch resulting in only a partial cancellation of the fields. This can be corrected by changing the spacing of the bunches within the train so that full cancellation is obtained, but since it is easier to generate a bunch train of equal spacing we choose to satisfy the above condition. Numerical simulations show that the 'symmetric, single wake' condition is met when the bunch length satisfies

$$
\sigma / \lambda_{0}=0.2
$$

For a given $R_{0}$, the transformer ratio is maximized if the conditions specified in Eqn. 2, Eqn. 3, and Eqn. 4 are satisfied. This means that if $R_{0}=1$, then the fastest $R_{n}$ can increase is $R_{0}=1, R_{1}=2, R_{2}=3, R_{3}=4$, etc. However, if $R_{0}=2$, then $R_{0}$ could increase as $R_{0}=2, R_{1}=4, R_{2}=6, R_{3}$ $=8$, etc. Therefore, since our goal is to maximize $R_{n}$ we desire $R_{0}=2$. From the previous section we know that $R_{0}$ $=2$ when,

$$
\sigma / \lambda_{0}=0.25
$$

\section{EXPERIMENTAL DESIGN}

The AWA facility [6] consists of two photoelectron sources operating at $1300 \mathrm{MHz}$ (or $\lambda_{\mathrm{rf}}=236.10 \mathrm{~mm}$ ) for the purpose of exciting and probing wakefields. Using the RBT algorithm and the details of the AWA facility we designed a bunch train and structure capable of achieving $R>2$. The RBT is generated by optically splitting the laser pulse and controlling the relative intensity and separation of the individual pulses. Details about RBT generation and the technique for measuring $R>2$ is given in Ref. 4.

\subsection{Bunch Train Design}

Since the AWA facility provides a bunch length of $\sigma=$ $4 \mathrm{~mm}$, then Eqn. 4 implies the optimum wavelength $\lambda_{0}=$ $20 \mathrm{~mm}$. However, since the operating frequency of the facility is $\mathrm{f}_{\mathrm{rf}}=1300 \mathrm{MHz}\left(\lambda_{r f}=230.61 \mathrm{~mm}\right)$ then the half integer condition of Eqn. 2 with $\mathrm{m}=10$ implies $\mathrm{f}_{0}=13625$ $\mathrm{MHz}\left(\lambda_{0}=230.61 \mathrm{~mm} /(10+0.5) \approx 22 \mathrm{~mm}\right)$. In this case $\sigma / \lambda_{0}=0.18$ - still nearly satisfying 'the symmetric, single wake' condition of Eqn. 4. According to the algorithm given above, we construct a train of 4 electron bunches of charge magnitude $Q_{0}=1 \mathrm{nC}, Q_{1}=3 \mathrm{nC}, Q_{2}=5 \mathrm{nC}$ and $Q_{3}=7 \mathrm{nC}$.

Detailed MAFIA simulations show that to get the maximum transformer ratio, we have to make to small adjustments to the structure and bunch train spacing. First we adjust the operating frequency, slightly, from 13625 $\mathrm{MHz}$ to $13625 \times 1.004=13679.5 \mathrm{MHz}\left(\lambda_{0}=21.92 \mathrm{~mm}\right)$ so that spacing between the first and second bunch is $d_{l}=\lambda_{0}$ 
$=230.16 \mathrm{~mm}$. Second, the separation between first bunch and third $d_{2}=(2+0.001) \lambda_{0}$ and the separation between first bunch and fourth $d_{3}=(3+0.003) \lambda_{0}$. Or, $d_{1}=$ $230.610 \mathrm{~mm}, d_{2}=461.243 \mathrm{~mm}$ and $d_{3}=692.522 \mathrm{~mm}$.

\subsection{Disk-loaded Structure Design}

A TW disk-loaded structure operating in the $2 \pi / 3$ mode with an operating frequency of $\lambda_{0}=13.6795 \mathrm{GHz}$ was designed [5] using Superfish. To achieve the above operating frequency the cavity dimensions [Fig. 3] were found to be $\mathrm{t}=2 \mathrm{~mm}, \mathrm{a}=5 \mathrm{~mm}, \mathrm{~b}=9.653 \mathrm{~mm}$, and $\mathrm{d}=$ $7.334 \mathrm{~mm}$.

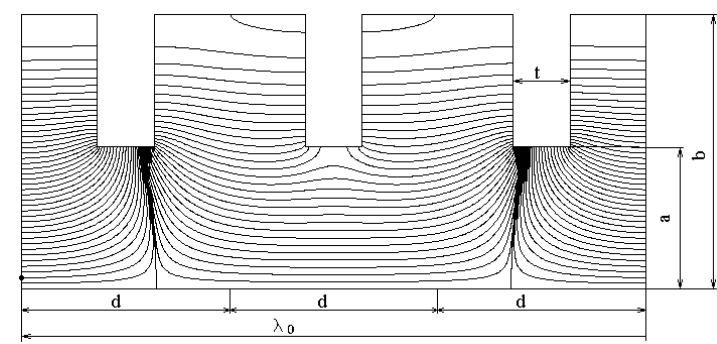

Figure 3: $2 \pi / 3 \mathrm{TW}$ disk-loaded structure designed with Superfish. $\mathrm{t}=$ Disk thickness; $\mathrm{d}=$ cell length; $\mathrm{a}=$ inner radius; $b=$ outer radius.

By using the geometry from Superfish, we designed a 39 cavity plus 2 half-cavity structure [fig. 4] for the RBT Experiment. This cavity has been constructed and it will be installed into the beamline in the near future.

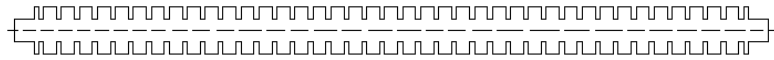

Figure 4: The 40 cavity structure to be used for the

Transformer Ratio Enhancement experiment. The wakefield is calculated by using the Mafia 2-D module with 300,000 mesh points.

We used Mafia to calculate the net wakefield [Fig.5] produced in this structure by the ramped bunch train of four bunches described above. Notice that each bunch experiences the same decelerating wakefield as in Fig. 1b, but the accelerating wakefield behind each bunch grows progressively larger. The transformer ratio, $R$, is found by dividing the maximum accelerating field behind the bunch train by the maximum decelerating field experienced by an individual bunch.

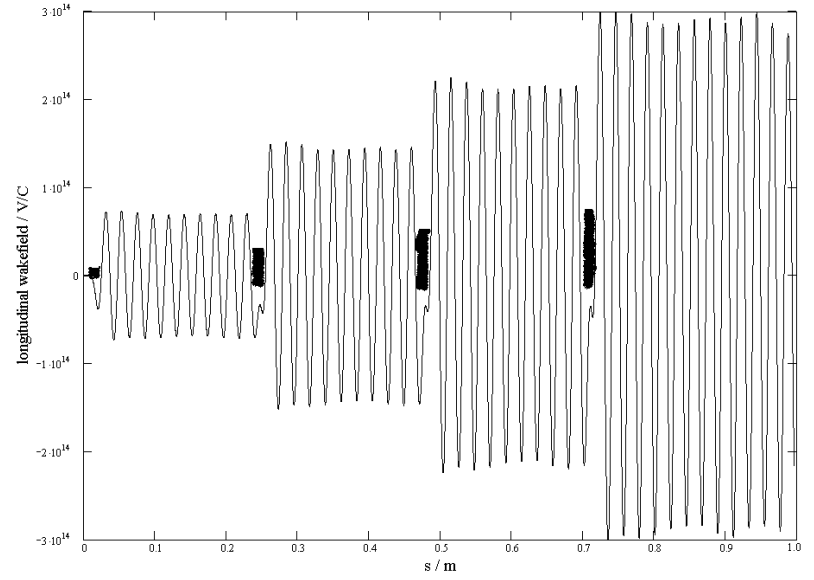

Figure 5: The resultant wakefield produced in the designed structure by a train of 4 electron bunches (shown in black) each of length $4 \mathrm{~mm}$. Charge magnitude $Q_{0}=1$

$\mathrm{nC}, Q_{1}=3 \mathrm{nC}, Q_{2}=5 \mathrm{nC}$ and $Q_{3}=7 \mathrm{nC}$. Bunch separation $d_{1}=\lambda, d_{2}=2.001 \lambda, d_{3}=3.003 \lambda$, where $\lambda=$ $230.11 \mathrm{~mm}$. The Transformer Ratio $R=25 / 3.8=6.6$.

\section{SUMMARY}

From [4], we use the RBT method for achieving a transformer ratio $(R)$ greater than 2 in any collinear wakefield accelerator and the algorithm for designing an RBT experiment. Using this, an experiment is planned to be performed at the AWA facility to measure $R>2$. In order to test the transformer ratio enhancement concept, we have designed an experiment. Using the RBT algorithm, we designed a train of 4 drive bunches length 4 $\mathrm{mm}$ and charge magnitude $Q_{0}=1 \mathrm{nC}, Q_{1}=3 \mathrm{nC}, Q_{2}=5$ $\mathrm{nC}$ and $Q_{3}=7 \mathrm{nC}$. The separation between the bunches is $d_{1}=\lambda, d_{2}=2.001 \lambda$ and $d_{3}=3.003 \lambda$, where $\lambda=230.11$ $\mathrm{mm}$. The geometry of the $13679.5 \mathrm{MHz}$ disk-loaded structure was designed with Superfish. The wakefields of the structure were calculated with Mafia. The Transformer Ratio in this experiment is expected to be 6.6 for the above bunch train. The structure has been constructed and it will be installed into the beamline in the near future and experiments will subsequently ensue.

\section{REFERENCES}

[1] P.B. Wilson, Proc. of the $13^{\text {th }}$ SLAC Summer Inst. on Particle Physics, SLAC Report No. 296, p. 273, E. Brennan ed., (1983)

[2] K.L. Bane, P. Chen, P.B. Wilson, IEEE Trans. Nucl. Sci. 323524 (1985).

[3] P. Schutt, T. Weiland, V.M. Tsakanov, On the Wake Field Acceleration using a Sequence of Driving Bunches., Nor Amberb Conf. Proc., Armenia, (1989)

[4] J.G. Power, M.E. Conde, W. Gai, A. Kanareyken, R. Konecny, and P. Schoessow, To be published in AAC 2000 Proceedings

[5] M. E. Conde, W. Gai, R. Konecny, X. Li, J. G. Power, and P. Schoessow Phys. Rev. ST Accel. Beams 1, 041302

[6] http://gate.hep.anl.gov/awa/awa/docs/WF-203-RBT9511.pdf 